\title{
A Literature Review of Root-End Filling Materials
}

\author{
Priyanka.S.R, Dr.Veronica \\ (Saveetha Dental college, Saveetha University, India) \\ (Department of Conservative dentistry and Endodontics, Saveetha Dental college, Saveetha University, India)
}

\begin{abstract}
Surgical endodontic therapy is done when non-surgical endodontic treatment is unsuccessful. Rootend resection is the most common form of periradicular surgery. The procedure involves surgical access or osteotomy to expose the involved area, root-end preparation, root-end resection, periradicular curettage and placement of a suitable root-end filling material. This article reviews the effectiveness of various available, time-tested and newer root-end filling materials including their biocompatibility, sealing ability, anti-bacterial effects and capacity to stimulate regeneration of normal periodontium.
\end{abstract}

Keywords: endodontic surgery, filling, retrograde, review, root-end

\section{Introduction:}

The goal of endodontic therapy is to hermetically seal all pathways of communication between the pulpal and periradicular tissues. A mandatory requirement of root canal therapy is that the obturation and restoration of the tooth must seal the root canals both apically and coronally to prevent leakage and percolation of oral fluids and to prevent recontamination of disinfected canals. Apicoectomy (apicectomy / root-end resection) with retrograde obturation is a widely applied procedure in endodontics, when all efforts for the successful completion of orthograde endodontic therapy have failed [1]. Failure of non-surgical endodontic therapy or non-surgical endodontic retreatment indicates the need for endodontic surgery to save the tooth. As most endodontic failures occur as a result of leakage of irritants and microbes from infected root canals, the root-end filling material must provide an adequate apical seal and be biocompatible. Its anti-bacterial effects and ability to stimulate regeneration of the periodontium will accelerate the healing process and reduce the incidence of failures. Root-end materials must be non-toxic, non-irritant, radio-opaque and non-corrosive. In addition to this, it should also be dimensionally stable, and easy to handle. Many materials have been used as root-end filling materials. These include amalgam, gutta percha, zinc-oxide eugenol cements ( IRM, Super-EBA), MTA, Glass ionomer cements, composite resins, compomers, diaket, Biodentine, Ceramicrete, Bioaggregate, EndoSequence, etc.

\section{Gutta Percha:}

Gutta Percha was introduced by Bowman in 1867. It is the most popular and most commonly used core filling material in endodontics. It is a trans-isomer of polyisoprene, existing in alpha and beta crystalline forms.[2]. Friedman described its composition as consisting of $20 \%$ gutta-percha matrix, $60 \%$ zinc oxide filler, $11 \%$ heavy metal sulphates as radioopacifiers and $3 \%$ waxes as plasticizers.[2]. Gutta percha is known to have a poor sealing ability as it has to be used with a sealer during root canal obturation. Use of gutta-percha as a rootend filling material is no longer recommended owing to the advent of newer materials with significantly enhanced properties.

\section{Amalgam:}

Silver Amalgam has been in use as a root-end filling material since 1884 (Farrar 1884 in Gutmann and Harrison 1999)[3]. Amalgam remains a standard to which all other materials are compared. It is readily available, easy to handle and manipulate, and is radio-opaque. But, there are many disadvantages that have been recognized which include marginal leakage, secondary corrosion, moisture sensitivity, and safety issues due to mercury toxicity [4]. A study by Tanzilli et al suggested that amalgam gives a poor seal when used as a retrograde filling material. But, the use of amalgam with 4-methacryloxy trimellitate anhydride bonding agent is shown to reduce microleakage. [5]. Electrochemical corrosion products of amalgam were reported to be responsible for failure of root-end fillings.[5]. A study of tissue response to various root-end filling materials done by Chong et al in 1997 showed that all roots filled with amalgam showed moderate or severe inflammation. Scattering of excess amalgam particles during placement of the root-end filling can lead to corrosion of the implanted material and cause unsightly amalgam tattoos [6]. Many clinical studies have shown poor outcomes with amalgam root-end fillings and amalgam can no longer be considered as the ideal root-end filling material[6]. 


\section{Polycarboxylate cements:}

Introduced by Smith in 1968, Zinc polycarboxylate cement consists of a powder which contains zinc oxide, magnesium oxide, bismuth and aluminium oxides, stannous fluoride. The liquid is and aqueous solution ofpolyacrylic acid or a copolymer of polyacrylic acid with other carboxylic acids like itaconic acid. The cement is believed to act with calcium ions through carboxyl groups on the surfaces of enamel and dentin. The bond strength to enamel is greater than in dentin. The sealing ability of polycarboxylate cement, as shown by Barry et al using dye penetration methods is inferior to amalgam [7].

\section{Cavit:}

Cavit was introduced as a temporary filling material made of zinc oxide and zinc sulphate without eugenol. Evaluation of the sealing ability of amalgam, Cavit and glass ionomer cement was done to reveal that Cavit had a better seal than amalgam but the seal was inferior to that of amalgam[8].

\section{Zinc Oxide Eugenol Cements:}

Zinc-oxide eugenol cements are among the most commonly used and recommended root-end filling materials. ZOE cements, in order to improve their physical properties was subjected to various modifications.

\subsection{Super EBA:}

Here, there is a substitution of part of the eugenol liquid with ortho-ethoxybenzoic acid (EBA) and addition of alumina to the powder. Super-EBA was developed in the 1960's, it was originally manufactured by Staines in England. This contained a powder component with $60 \%$ zinc oxide, $34 \%$ silicon dioxide, $6 \%$ natural resin, and a liquid component with $62.5 \%$ ortho-ethoxy benzoic acid and $37.5 \%$ eugenol. The Harry.J.Bosworth Co. used the same liquid component and replaced the silicon dioxide in the powder with $34 \%$ alumina [9]. Super EBA shows high compressive strength, high tensile strength, neutral $\mathrm{pH}$ and low solubility. A comparative study of the solubility of some root-end filling materials done by Poggio et al in 2007 showed that IRM, SuperEBA and MTA showed no signs of solubility in water. It has also been shown to have good sealing characteristics. An in vitro microleakage study done by Yaccino et al in 1999 suggested that fast set or regular set super-EBA used in various consistencies may be acceptable as root-end fillings. It adheres well to tooth structure even in moist conditions. Reports show a good healing response to super-EBA with minimal chronic inflammation at the root apex. But, super-EBA is radioluscent and technique sensitive. The eugenol content of super-EBA may be a source of irritation to the tissues.[4].

\subsection{IRM:}

IRM is zinc oxide eugenol cement modified by addition of $20 \%$ polymethyl methacrylate by weight to the powder. the effect of IRM as a root-end filling placed in teeth prior to replantation was observed by Pitt Ford et al in 1994 and the tissue response was found to be less severe than that to amalgam. Eugenol in IRM may have an affinity for poly methyl methacrylate which reduces its release into the tissues, thereby reducing the cytotoxicity.[6]. Zinc oxide eugenol cements, IRM and super-EBA were analysed for their release of zinc and eugenol by Al-aseed et al in 2008. Eugenol release from IRM by this leached component analysis was obviously higher than from Super-EBA because of the comparatively higher content of eugenol. But this higher release of eugenol did not increase its cytotoxicity; super-EBA was more toxic. So, the release of zinc may be the main cause of toxicity due to ZOE cements. IRM was shown to have a better seal than amalgam or super-EBA. IRM showed good anti-bacterial activity against S.aureus, E.faecalis, P.aeruginosa.[10].

\section{Mineral Trioxide Aggregate:}

Gray Mineral trioxide aggregate (MTA) was developed at Loma Linda University, California by Torabinajed \& co-workers in 1993. MTA has shown excellent seal and hard tissue repair compared with other root-end filling materials. The main components in MTA are tricalcium silicate, tricalcium aluminate, tricalcium oxide, silicate oxide. Bismuth oxide has been added to the powder to make it radio-opaque. The powder is composed of hydrophilic particles that set in the presence of moisture. Hydration of the powder forms a colloidal gel that hardens [4]. According to a clinical study done by Chong and Pitt ford in 2003 comparing MTA and IRM, the use of MTA showed a higher success rate. MTA has shown promising results due to its good sealing properties, bioactivity, and potential to stimulate cementogenesis. The main advantages of MTA are its biocompatibility and its osteogenic and regenerative potential [11].MTA has been demonstrated to have better anti-bacterial properties against E.faecalis, S.aureus and P.aeruginosa compared to other materials. In addition, MTA has better anti-bacterial activity when used after mixing with $0.12 \%$ chlorhexidine [12]. In a study done to compare sealing abilities of white and gray MTA when mixed with water and $0.12 \%$ chlorhexidine which showed no differences in sealing abilities.this shows that CHX does not compromise the sealing effect of MTA [13]. The use of MTA has been shown to induce cementum formation and periodontal regeneration with induction of least amount of inflammation. Tissue culture experiments suggest that MTA 
induces cementogenesis, permitted cementoblast attachment and growth. MTA shows no toxic effects on cells and is reported to cause an increase in cell proliferation and released calcium in high amounts. [14]. A disadvantage is its slow setting and less resistance against washing out during placement.

\section{Glass Ionomer Cement:}

Glass ionomer cement was introduced as a new restorative material in the early 1970s. They are based on the reaction of ion-leachable, acid soluble calcium fluoro aluminosilicate glass particles with polyalkenoic acid. They possess adhesive properties forming a chemical bond with dentin, and have a significant fluoride releasing property. Resin-modified glass ionomer cements were first described by Antonucci et al to improve physical properties and handling characteristics. They contain a monomer such a Hydroxyethyl methacrylate (HEMA) or bisphenol-A-glycidyl methacrylate (bis-GMA) along with a photo-initiator such as camphoroquinone. Glass ionomer cements induce an intense inflammatory response which resolves and the inflammation is replaced by bone. Silver-reinforced glass ionomer cements were also tried which showed a good tolerance but it released more amounts of silver which caused discolouration similar to amalgam and the corrosion products were cytotoxic.[6]. Generally, glass ionomers are slow setting, awkward to handle and are very sensitive to moisture contamination. Using them in a surgical field only amplifies this problem. Resinmodified glass ionomers - Vitrebond as a potential root-end filling material improved the handling properties and had a good adaptation and sealing ability. The sealing ability of light-cured glass ionomer cements was significantly better than that of amalgam and also slightly better than conventional glass ionomer cements[6]. In vitro studies showed a good antibacterial effect and low cytotoxicity. In a comparative study of tissue response to amalgam, Vitrebond and Kalzinol in an experimental model of infected root canals, Vitrebond and Kalzinol showed a tissue response considerably more favourable than amalgam root-end filling, even in the short term. After one week, the overall best tissue response was to Vitrebond, followed by Kalzinol[6]. These are easier to handle and light-curing helps control the setting reaction, but, maintenance of a dry field during placement still presents a challenge as it may interfere with the dentin bond.

\section{Composite Resins:}

Use of composite resins along with dentin bonding agent is also used to produce a leak-resistant seal. Rud et al have shown excellent long term clinical success with Retroplast composite resin root-end fill and Gluma dentin bonding agent. But, presence of a dry field during placement is important. Conventional composite resins contain a polymerizable organic matrix, inorganic fillers and a silane coupling agent. TEGDMA, bis-GMA and UDMA have been detected in aqueous extracts [15] and formaldehyde can liberate over a long time period [16]. These components may be the reason why the material exhibits highly antibacterial effects against P.gingivalis, P.intermedia, P.endodontalis, F.nucleatum [19].

Enamel matrix derivatives (EMD) coated on surfaces of root dentin is known to promote periodontal regeneration. Periapical biopsies of teeth with composite resin retrograde fillings have shown deposition of cementum and reformation of periodontal ligament over the resin fillings [17]. An experiment done to evaluate the adherence of enamel matrix derivatives on root-end filling materials was done to compare amalgam, IRM and Composite resin. High amounts of EMD were found to adhere to the composite resin. This could be an explanation for the periodontal regeneration seen with composite resin fillings [18].

\section{Compomers:}

Compomers which are poly-acid modified composite resins were developed to combine the fluoride releasing property of glass ionomer cements with the mechanical properties of composite resins. The setting reaction is an addition polymerization which is light-initiated, similar to composite resins. The monomer contains acidic functional groups and the material sets via a free radical polymerization reaction. It does not bond to tooth structure like glass ionomer cement but need a bonding agent like composite resins.[19]. Compomers may release fluoride in the first few days after polymerization due to the presence of ion-leachable glass fillers, similar to glass ionomer cements. In a study done to compare silver amalgam and compomer as retrograde filling materials, a histological study of the samples involving retrograde filling with amalgam in animal models revealed average biocompatibility with limited bone formation and moderate inflammation. The compomer group showed greater inflammation showing its low biocompatibility but also showed greater root cementum growth. [20]. Other studies with an in vivo intraosseous implantation in rabbit showed that it has a good biocompatibility as Super-EBA. Gingival tissues appear to adhere to the material, allows fibroblasts to reform around the root apex in which compomer root-end filling is placed.[21]. Dyract has been shown to have good anti-bacterial effects against P.gingivalis, P.intermedia, P.endodontalis and F.nucleatum. the release of residual monomers and additives after polymerization may be the reason for the anti-bacterial effect. The results of an electrochemical study of the sealing ability of super-EBA, MTA and Dyract-flow showed that the sealing ability of Dyract-flow is equal to that of super-EBA and MTA[22]. 


\section{Titanium screws:}

A study of titanium screws as retrograde fillings was done to compare it with amalgam. Bacterial penetration was seen readily on the first day in the amalgam fillings but bacteria penetrated the titanium screw seals after 2 to 7 days. Titanium screws appeared to produce a tighter seal than amalgam.[23].

\section{Diaket:}

Diaket, which is normally used as a root canal sealer has been used as a root-end filling when mixed to a thicker consistency. As a root canal sealer, it was shown to be tolerated by the tissues. Stewart in 1958 showed that Diaket is impervious to methylene blue dye and does not dissolve or absorb in the presence of periradicular tissue and fluids. As a root-end filling, diaket is shown to have superior sealing qualities when compared to amalgam [24]. Diaket also shows a good healing response characterized by bone apposition, reformation of periodontal ligament and deposition of new cementum [25].

\subsection{Ceramicrete :}

\section{Newer Materials:}

Ceramicrete is an inorganic phosphate ceramic binder material used to encapsulate radioactive and hazardous wastes. It is a self-setting phosphate ceramic that sets using an acid-base reaction to form a potassium magnesium phosphate hexahydrate ceramic matrix phase. Its mechanical properties were improved by adding calcium silicate whiskers to produce a phosphosilicate ceramic material [26]. A ceramicrete based dental or bone material was introduced which had hydroxyapatite powder and cerium oxide radioopaque fillers [27]. This material is biocompatible and radioopaque. The material is also known to release calcium and phosphate ions during setting.

An in vitro study was done to evaluate the Ceramicrete based material as a root-end sealing material. This study used a ceramicrete-based powder mixed with deionized water. This study showed that ceramicrete had a radioopacity similar to root dentin, and the sealing ability was higher compared to a SuperEBA and ProRoot MTA group. This excellent apical seal was attributed to its impervious nature and also the use of an acidic $\mathrm{MgH}_{2} \mathrm{PO}_{4} \cdot \mathrm{H}_{2} \mathrm{O}$ solution as a conditioner to remove the smear layer which is believed to have improved the adaptation of ceramicrete with the dentin. On immersion of the set ceramicrete material in a Phosphate containing fluid (PCF), there was formation of Dicalcium phosphate dihydrate (DPCD) or hydroxapatite on the surface. This is due to the reaction of calcium disilicate from the ceramicrete material with the phosphate from the PCF. Thus, ceramicrete shws potential bioactivity.[28].

A comparison of the root-end seal achieved using Ceramicrete, Bioaggregate and White MTA was done to study the prevention of glucose penetration. Both Bioaggregate and Ceramicrete showed similar sealing ability to MTA, with Ceramicrete showing significantly better results than Bioaggregate [29].

\subsection{Biodentine:}

Biodentine is a calcium silicate based material introduced in 2010 as a material for crown and root dentin repair treatment, repair of perforations, apexifications, resorption repaie and root-end fillings [30]. The main component is a highly purified tricalcium silicate powder that contains small amounts of dicalcium silicate, calcium carbonate, and a radioopaquer [31]. An in vitro study to compare the sealing ability of MTA, Calcium phosphate cement and Biodentine MTA showed the highest seal and the least dye absorbance. Biodentine showed a seal slightly less than MTA but, higher than Calcium phosphate cement[32]. The interfacial properties of dentin-biodentine interface were studied under microscope and tag-like microstructures were detected. The flowable consistency of Biodentine penetrates dentinal tubules and helps in the mechanical properties of the interface [31].

Investigation of the bioactivity of Biodentine, MTA and a new Tricalcium silicate cement revealed that all three cements allowed the deposition of hydroxyapatite on the surface. This shows that all three materials are bioactive [33].

\subsection{Bioaggregate:}

Bioaggregate is a new bioceramic root repair and root-end filling material composed of a powder component consisting of tricalcium silicate, dicalcium silicate, tantalum pentoxide, calcium phosphate monobasic and amorphous silicon oxide and a liquid component of deionized water.

A study investigated the cytotoxicity and the effect of Bioaggregate on Mineral-associated gene expression in osteoblast cells. Bioaggregate was shown to be non-toxic to osteoblast cells and it was also shown to enhance expression of genes for collagen type 1, osteopontin and osteocalcin, which are genes associated with mineralization in osteoblast cells [34]. In a study done to compare the cytotoxicity of ProRoot MTA and DiaRoot Bioaggregate, Bioaggregate showed a significantly better inflammatory reaction and foreign body reaction than the MTA group. Therefore, Bioaggregate is more biocompatible than MTA [35]. The effect of 
Bioaggregate and MTA on human pulp and PDL cell growth was determined byexamining the cells grown on this cement using a phase microscope. An inhibition zone was detected in the pulp and PDL cell culture grown with MTA. Bioaggregate showed no inhibition zone around the material. Bioaggregate was found to be nontoxic to human pulp and PDL cells [36].

Leal et al showed that the sealing abity of white MTA and Bioaggregate were similar in preventing glucose penetration, but slightly less than Ceramicrete. An in vitro comparative study of the sealing ability of Diadent Bioaggregate and other root-end filling materials (Gutta-percha, amalgam, IRM, White MTA) was done using methylene blue dye penetration technique. The results showed that microleakage was significantly less in Bioaggregate when compared to amalgam, IRM and White MTA [37]. Bioaggregate's excellent hermetic seal can be attributed to its nano-sized particles that adhere to the dentinal wall, and its hydrophilic nature.

\subsection{Endosequence}

Endosequence Root Repair Material (ERRM) is a new bioceramic material consisting of calcium silicates, monobasic calcium phosphate, and zirconium oxide [38]. ERRM is radioopaque, biocompatible and bioactive. Its high $\mathrm{pH}$ contributes to its antimicrobial activity [38]. The bioactivity was tested in a study by exposing the set material in phosphate-buffered saline. There was precipitation of apatite crystalline structures, which is indicative of its bioactivity [39]. ERRM has been shown to have negligible cytotoxicity and capability to induce cytokine expression similar to MTA [38].

\section{Conclusion:}

An ideal root-end filling material is still elusive because each of these above discussed materials have their own advantages and disadvantages. Currently, we cannot recommend an ideal root-end material as none of the materials available satisfy all of the desired qualities. The outcome of periradicular surgery can also be affected by factors other than insufficient apical seal or others.

\section{References:}

[1] Gutmann and Harrison, Gutmann's surgical endodontics, 1999.

[2] Grossman, Grossman's endodontic practice $12^{\text {th }}$ edition, 2010.

[3] R. Niedermann, J.N. Theodosopoulou, A systematic review of in vivo retrograde obturation materials, International Endodontic Journal, 36, 2003, 577-585

[4] Claudio Poggio, Marco Lombardini, Conti Alessandro, Rindi Simonetta, Solubility of Root-end filling materials: A comparative study, Journal of Endodontology, 33(9), 2007, 1094-1097.

[5] Anderson RW, Pashley DH, Pantera EA, Microleakage of amalgambond as retrograde fillings in endodontic therapy, Journal of Endodontology, (9), 1983, 551-553.

[6] B.S.Chong, T.R.Pitt Ford, S.P.Kariyawasam, Short term response to potential root-end filling materials in infected root canals. International Endodontic journal,(30), 1997, 240-249.

[7] Gene Norman Barry, Alan.G.Selbst, Erbert W.D'Anton, Richard M.Madden, Sealing quality of polycarboxylate cements when compared to amalgam as a retro-filling material, Journal of oral medical, oral surgery and oral pathology, 42(1), $1976,109-116$.

[8] Hatem A. Alhadainy, Van T. Himel, Evaluation of the sealing ability of amalgam, Cavit and Glass ionomer cement in the repair of furcation perforation, Journal of oral medicine, oral surgery and oral pathology, 75(3), 1993, 362-366.

[9] John.M.Yaccino, William.A.Walker, David.L.Carnes, William.G.Schindler, Longitudinal microleakage evaluation of Super-EBA as a root-end sealing material, Journal of Endodontics, 25(8), 1999, 552-554.

[10] Ayce Unverdi Eldeniz, Hasan Huseyin Hadimli, Hanife Ataoglu, Dag Orstavik, Antibacterial effect of Selected root-end filling materials, Journal Of Endodontology, 32(4), 2006, 345-349.

[11] William Philip Saunders, A prospective clinical study of periradicular surgery using Mineral trioxide aggregate as a root-end filling, Journal of Endodontology, 34(6), 2008, 660-664.

[12] Stowe TJ, Sedgley CM, Stowe B, Fenno JC, The effects of chlorhexidine gluconate $(0.12 \%)$ on the anti-microbial properties of tooth-coloured Pro-root MTA. Journal of Endodontology,(30), 2004, 429-431.

[13] Chung-Chih Lai, Fu-Mei Huang, You Chan, Hui-Wen Yang, Min-Sheng Huang, Ming-Yung Chou, Yu-Chao Chang, Antibacterial effects of resinous retrograde root filling materials, Journal of Endodontics, 29(2), 2003, 118-120.

[14] R.Al-Sa'eed, Ahmad S. Al-Hiyasat, Homa Darmani, The effects of six root-end filling materials and their leachable components on cell viability, Journal of Endodontology, 34(11), 2008, 1411-1414.

[15] Ferracane JL, Condon JR, Rate of elution of leachable components from composite, Dental Materials, (6), 1990, 282-7.

[16] Geurtsen W, Substances released from dental resin composites and glass ionomer cements, European Journal of Oral Sciences, (106), 1998, 687-95.

[17] Andreasen JO, Munksgaard EC, Fredebo L, Rud J, Periodontal tissue regeneration including cementogenesis adjacent to dentinbonded retrograde composite fillings in humans, Journal of Endodontology, 19, 1993, 151-153.

[18] Kamran Safavi, Reza Kazemi, Dudley Watkins, Adherence of enamel matrix derivatives on the root-end filling materials, Journal of endodontics, 25(11), 1999, 710-712.

[19] . John W.Nicholson, Polyacid-modified composite resins (compomers) and their use in clinical dentistry, Academy of dental materials, 2006, 968.

[20] Jordi Gargallo Albiol, Jose Manuel Aguirre Urizar, Cosme Gay Escoda. Med Oral Patol Oral Cir Bucal, 13(2), 2008, E133-137.

[21]. Dragoo MR, Resin-ionomer and hybrid-ionomer cements, Part II, Human clinical and histologic wound healing responses in specific periodontal lesions, International Journal of Periodontal and Restorative Dentistry, (17), 1997, 75-78.

[22] Dong Sung-park, suh-Jin Sohn, Tae-Seok oh, Hyun-Mi Yoo, Chan-Je Park, Soon-Ho Yim, Young-Kyoo Lee, Seung-Bum Kye, An electrochemical study of the sealing ability of three retrofilling materials, Journal of Korean Academy for Conservative Dentistry, 29(4), 2004, 365-369 
[23] Luomanen M, Tuompo H, Study of titanium screws as retrograde fillings using bacteria and dye, Scandinavian journal of dental research.

[24] Kodohiro G, A comparative of the sealing quality of zinc-free malgam and Diaket when used as a retrograde filling material, Hawaii Dental Journal (15), 1984, 8-9.

[25] Witherspoon DE, Gutmann JL, Analysis of the healing response to gutta-percha and Diaket when used as root-end filling materials in periradicular surgery, International Endodontic Journal, (33), 2000, 37-45.

[26] AS, Jeong SY, Lohan D, Elizabeth A, Chemically bonded phosphor-silicate ceramic, U.S.Patent \& Trademark office, 2006.

[27] Wagh AS, Primus C, Method method and product for phosphosilicate slurry for use in dentistry and related bone cements, US Patent No. 7,083,672, US Patent \& Trademark office, 2006.

[28] Kelvin C.Y. Tay, Bethany A.Loushine, Cindy Oxford, Rishi Kapur, Carolyn M. Primus, James L. Gutmann, Robert J.Loushine, David H. Pashley, Franklin R. Tay, In vitro evaluation of a Ceramicrete-based Root-end filling material, Journal of endodontology, 33(12), 2007, 1438-1443.

[29] F.Leal, G.De-Deus, C.Brandao, A.S.luna, S.R.Fidel, E.M.Souza, Comparison of the root-end seal provided by bioceramic repair cements and white MTA, International endodontic journal, 44, 2011, 662-668.

[30] Trond Bjorvik Osen, Ina Iselin Astrup, Carl Haavard Knutsson, Biodentine as a root-end filling, 2012.

[31] A.R.Atmeh, E.Z.Chong, G.Richard, F.Festy, T.F.Watson, Dentin-cement interfacial interaction: Calcium silicates and Polyalkenoates, Journal of dental research, 91(5), 2012, 454-459.

[32] Tulsi Sanghavi, Nimisha Shah, Ruchi Rani Shah, Comparative analysis of sealing ability of Biodentine and Calcium phosphate cement against MTA as a furcal repair material, National journal of integrated research in medicine, 4(3), 2013, 56-60.

[33] Camilleri J, Sorrentino F, Damidot D, Investigation of the hydration and bioactivity of radiopacified tricalcium silicate cement, Biodentine and MTA Angelus, Academy of Dent materials, 29(5), 2013, 580-593.

[34] Zhenglin Yuan, Bin Peng, Han Jiang, Zhuan Bian, Ping Yan, Effect of Bioaggregate on Mineral-associated Gene Expression in Osteob last cells, Journal of Endodontology, 36(7), 2010.

[35] Yusuf-Burak Batur, Gozde Acar, Yagmur Yalcin, Seckin Dindar, Hande Sancakli, Ugur Erdemir, The cytotoxic evaluation of mineral trioxide aggregate and bioaggregate in the subcutaneous connective tissue of rats, Medical oral Pathology Oral Cir Bucal, 18(4), 2013, 745-751.

[36] Choo-Ryung Chung, Euiseong Kim, Su-Jung Shin, Biocompatibility of bioaggregate cement on human pulp and periodontal ligament derived cells, Journal of Korean academy of conservative dentistry, 35(6), 2010, 473-478.

[37] El Sayed MA, Saeed MH. In vitro comparative study of sealing ability of Diadent Bioaggregate and other root-end filling materials, Journal of conservative dentistry, 15(3), 2012, 249-252.

[38] N.Shokouhinejad, M.H.Nekoofar, H.Razmi, S.Sajadi, T.E.Davies, M.A.Saghiri, H.Gorjestani, P.M.H.Dummer. Bioactivity of Endosequence Root Repair Material and Bioaggregate, International Endodontic Journal, 45, 2012, 1127-1134.

[39] Maria Ciasca, Anita Aminoshariae, Thomas Montagnese, Andre Mickel, A comparison of the cytotoxicity and Proinflammatory cytokine production of Endosequence Root Repair Material and ProRoot Mineral Trioxide Aggregate in Human Osteoblast Cell Culture using Reverse Transcriptase Polymerase Chain Reaction, Journal of Endodontics, 2012. 